\title{
In-situ Study of Seating Static Comfort in Passenger Vehicles
}

\author{
Umi Salmah Seraj, Mohd Farid Aladdin ${ }^{1, *}$ \\ School of Engineering, Faculty of Built Environment, Engineering, Technology and Design, \\ Taylor's University, Malaysia.
}

\begin{abstract}
In today's automotive market, comfort is huge selling point of a vehicle. Priority is given by buyers to how comfortable a seat feels during purchase decisions. The measure of comfort is harmonious mix of many aspects such as human ergonomics and physiological factors. However, a gap still exists between objective and subjective measures due to lack of emphasis by past researchers. This is particularly obvious in the lumbar support feature that has still not been able to address the health problems related to driving. This project focuses on bridging the gap by giving users the ability to define true preferred posture in realistic settings. This is done by the creation of a apparatus that allows users to individually manipulate the seat contour for optimum support in more segments than just lumbar area. The experiment is performed in 3 parts, where in each part different segments of the apparatus are manipulatable (lumbar segment, sacral \& thoracic segment, and all segments). Sixty human subjects' statistics are recorded (gender, age, BMI and height) and the subjects are palpated to locate internal joints. These joints are marked and postural angles between them are measured using a goniometer. In each seat configuration, the angles are measured and a comfort rating is taken to be compared. It was found that the posture angles are different among the 3 experiments, and there is a change in comfort felt. Some human factors have also been proven to contribute heavily to angles chosen by occupants.
\end{abstract}

\section{Introduction}

The comfort facility of a seat is now as equally important as the functional and aesthetic aspects of automobiles for consumers to have an enjoyable ride [1]. The feeling of comfort has been collectively agreed on as [2]: a rise of a subjectively-defined personal character; exaggerated by physical, physiological, psychological characters; and a response to the environment. Normally the term comfort is more appropriately defined and studied as the absence of discomfort as it is easier to quantify discomfort than to measure comfort [2]. There is a large body of literature devoted to the study of automotive seating comfort, likely due to the economic costs associated with discomfort and injury in the motor-vehicle workplace. Studies have shown that low-back pain and serious conditions like spinal disc herniation worsen with increased driving hours [3], thus requiring ergonomic interventions in the early designing stages.

\footnotetext{
${ }^{1}$ Corresponding author: MohdFarid.Aladdin@taylors.edu.my
} 
The two categories in ergonomic criteria assessment studies are the physiological and anthropometric factors [4]. Due in large part to Akerblom's work, anthropometric studies have long been considered a key aspect of comfortable seating, repeatedly verified by Parkinson and Reed [5], Ziolek et al., [6] and Seitz et al., [7]. Designers aim to ensure that a wide range of people can fit and utilize the seat, commonly adapting the constraining anthropometric values of 5 th percentile female and 95 th percentile male for corresponding seat dimensions. However, the identification of posture based on anthropometry is difficult because people with similar body dimensions have proven to assume completely different positions [8]. Basic demographic information (e.g. BMI, age, etc.) is usually included to understand its effects on and classify the varying assumed position.

Examples of physiological factors on the other hand are: objective measurement using pressure distribution $[9,10]$, vibration analysis [11,12], surface electromyography $[13,14]$ and temperature at seat (On the other hand, the subjective measurement methods often use a rating scale and a questionnaire that elicits a rating for each of the defined seat regions in the experiments. Most research involves the changing of a critical aspect of the seat to form various iterations around which the research can be conducted and subjective comfort relationships studied. Some previous experiments define the subjective regions based on the part of the body that are interfaced with the cushion (upper back, middle back, lower back, etc.) [2] while other researchers tend to identify regions on the surface area of the seat (e.g. seat back - upper/mid/lower).

However, ergonomics criteria related to physiology have also recently come under scrutiny. Reed et al. described the automobile seat designer's dilemma of prescribing a physiologically suitable seated posture while accommodating a driver's preference, reasoning that prescribed postures sometimes compromise actual comfort. An important finding by Reed et al. vitally highlighted the incompatibility between the common practice of designing seatbacks to induce a large degree of lumbar lordosis and satisfying occupantselected spinal configurations (which are more kyphotic for some). Lordosis is associated with lower pressure in the lumbar intervertebral discs compared with more flexed (flat or kyphotic) lumbar spine postures, therefore regarded as more comfortable. Schneider verified this incompatibility in a follow-up study. Kolich et al. came to a similar conclusion.

Due to the varying preferences, comfortable accommodation in the lumbar region is thought to be best achieved through adjustability. However, evaluation of market products confirm that existing lumbar supports do not function as well as intended even with a wide range of adjustments. Yun Choi et al. found that even the recommended maximum prominence from past studies produced insignificant restoration of lordosis, while causing additional discomfort due to increased pressure concentrations and peaks at the lumbar area from firm padding. Warrow et al. described the conflict between the recommended heights of the support verses the large variability in occupant lumbar spine configuration, highlighting the placement issue of the support. More importantly, Eby discovered that the task of driving constrains postures to a great extent and prevent occupants from selecting lordotic postures that may cause them difficulty in vision and manoeuvrability, particularly those with extreme body proportions. This conclusion was reached by many other researchers.

The discrepancy in recommendations has proved to be difficult task for designers to interpret and integrate into current seats. A more recent discovery in seating is the appropriateness of sacral + thoracic support over lumbar support for seating comfort. Nairn, 
B.C found that sacral support will stabilize the pelvis in the proper neutral position, and lower thoracic support will apply an extension force that will result in elevation of the sternum and optimal curvature of the spine, restoring the proper axial/neutral relationship of the thorax and pelvis. This finding has been incorporated into many industrial products (e.g.: office chairs, massage equipment, wheelchairs, etc.) Latest seat configurations in luxury vehicle brands have also somewhat developed supports in these regions, although no standardization has been set due to lack of experimentation and data.

As stated by Hanson et al., it is not sustainable to rely on old recommendations when competing on a changing market. Therefore, it is necessary to study how much improvement the sacral and thoracic supports bring to the comfort of users in a passenger vehicle. With no current method to do so, an apparatus must be constructed to allow the study of different supports and the corresponding postures, and evaluate the differences in comfort rating felt by the passengers. Joint postural angles can be used to compare preferred postures as they are quantifiable and easily compared to one another, and verifiable with existing data where comfort feelings are not. It is also necessary to study the human factors that affect the chosen posture, which can be done by classification of the passengers' physical characteristics. Understanding this is important for future prediction, modelling and experimentation.

Therefore, the objectives of this study aligned to the theoretical framework and arising problems are: 1) To create an apparatus that allows drivers to define their own preferred posture in realistic settings. 2) To identify the potential differences between existing and preferred posture in terms of postural angles, as well as the resulting difference in comfort. 3) To evaluate the human factors that affect the outcoming preferred posture the most in terms of postural angles.

\section{Methodology}

\subsection{Design of Support Apparatus}

There are two important considerations for this artefact, which are:

a) adaptability to the adjustment preferences of a large variety of subjects; and

b) adherence to current seating contour constraints (lateral support).

The apparatus is only capable of forward extension (support prominence). By having separate adjustable segments at different fixed heights, the intended height of the prominence can also be somewhat varied. It has a maximum adjustment of $45 \mathrm{~mm}$ to accommodate any extreme postures. The anthropometric values used to ensure a wide fit was taken between the 5th and 95th percentile of Malaysian drivers. The sitting shoulder height for 95th percentile male $(645.55 \mathrm{~mm})$ is chosen to allow subjects in that range to make adjustments in the upper back area. The width should also be according to the 95th 
percentile male $(480.00 \mathrm{~mm})$, however due to current seats already having lateral supports, the flatback was set to be $280 \mathrm{~mm}$ which is the average width of the flat surface. The curvature was measured at $80 \mathrm{~mm}$ radius for most vehicles, which will be the minimum distance of the supports from the flatback to not be affected by them.

The apparatus has 3 main parts, which are the flatback, the jacks and the supports. To keep within the project's budget, most materials are obtained free or at a lower cost from associates. The jacks are made of aluminium links, steel left-hand/right-hand male threaded rods, steel left-hand (LH) and right-hand (RH) female nuts, steel left-hand and right-hand female hex coupling nuts and steel flat plates. The flatback is made of a wooden board, with fabric to cover the wood and avoid damage to car seats. The supports utilized armrest cushion for wheelchairs. An issue was the sizing, as the longest armrests available were 14 inches $(355.6 \mathrm{~mm}$ ) which is $125 \mathrm{~mm}$ shorter than the intended length. This might affect the comfort felt by larger occupants. The width of the armrest was 2.25 inches $(57.15$ $\mathrm{mm}$ ), making the vertical distance between the centres of each cushion when stacked lengthwise also at least $57.15 \mathrm{~mm}$. This value is close enough to the common $50 \mathrm{~mm}$ height interval in many researches.

The tests performed to ensure the prototype met the following requirements were:

a) Constraint fitting - the product needs to fit to the seat constraints.

b) Comfort feel - the product should not cause unnecessary discomfort to the subject as it diminishes the purpose of the study.

c) Versatility - the product's adjustability should be able to conform to the preference of a variety of subjects.

d) Stability - the product need to be sturdy and stable under the weight of subjects.

The most challenging aspect during testing was stabilizing the prototype. The weight caused a resistance force on the prototype that halted its expansion capability because the links were not strong enough on their own to support the occupant. The expansion of the prototype was not uniform on both sides and a bending moment was being produced that made the jack deflect slightly downwards when expanded. Two alternatives were finalized to address these issues: scissor mechanism or support block. The support block solution was chosen as the solution as the alternative was too time-consuming and its outcome unpredictable. The block idea involves including an expandable hollow block in the centre of the jack to direct most of the weight exerted on the cushion onto it. The strong opposing forces of the block will be able to balance out the weight of the occupant, and it's structure will ensure both sides expand/contract simultaneously. Deflection will also no longer occur as the block ensures only linear movement as according to its path. Figure 1 shows the completed prototype and apparatus 

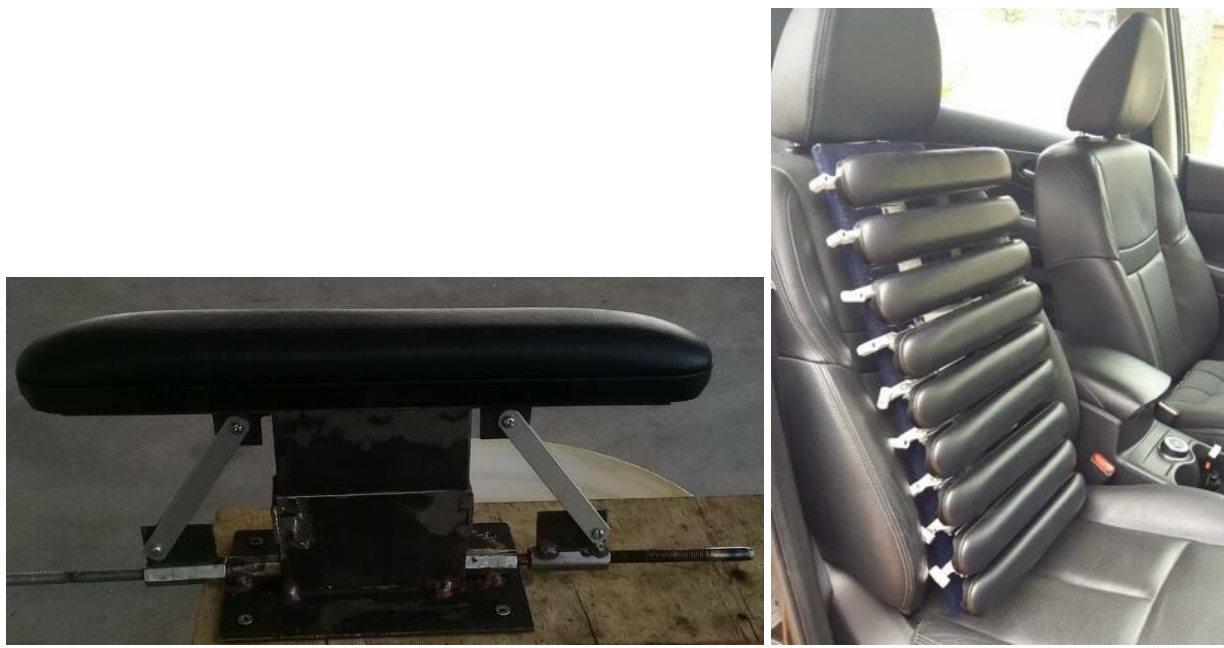

Figure 1. First successful prototype (left); completed apparatus tested in vehicle (right)

\subsection{Postural Study}

There are many ways to represent a vehicle occupant's posture including body landmarks, external body contours and segment linkage models. The chosen method is in terms of orientations (joint angles) of body segments. In the torso, the lumbar, thorax and cervical regions of the spine are each represented by a single segment instead of a singular torso segment to clearly represent the intra-subject posture changes corresponding to change in backrest supports as shown in Figure 2. Additional segments would provide better illustration, however since most studies have utilized these three-segment torso division, it would be easier to compare the data with verified value. Palpated surface landmarks shown in Figure 3 of the spinous process will be used to identify the joint locations that define the linkage.

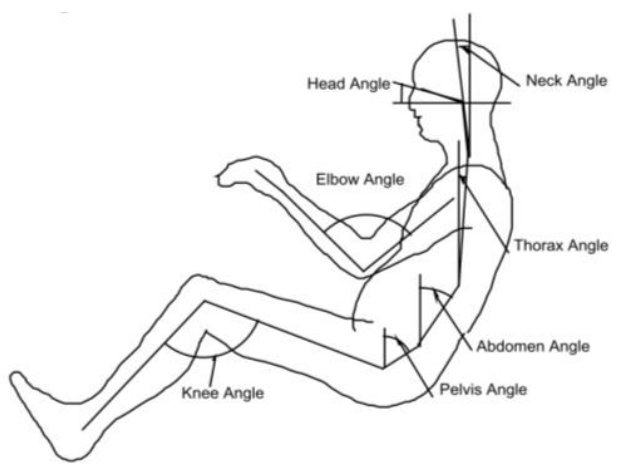

Fig. 2. Segment joint angles [15] 


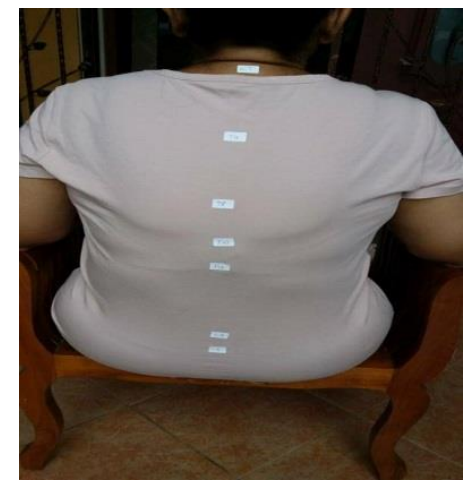

Fig. 3. Identification of palpation points

\subsection{Landmark-to-Joint Transformations}

The landmark-to-joint transformation methods used here are mostly based on the data analysis and summary presented by Reed, who referred his work from several sources, including a three-volume publication by Robbins. Robbins used external landmark data to estimate the internal joint locations of three categories of humans (large male, mid-sized male and small female) from experimentation and previous sources. The locations of joints relative to the surface landmarks are calculated using simple linear scaling relationships. Ultimately, the scaling approach for all subjects uses the midsize-male data as the reference geometry because the underlying data on which the landmark-to-joint transformations are based relied exclusively on male subjects, and because there are very minor differences between each category.

\begin{tabular}{|l|l|}
\hline \multicolumn{1}{|c|}{ Joint } & \multicolumn{1}{c|}{ Landmarks } \\
\hline Upper Neck (atlanto-occipital) & Infraorbitale, Tragion \\
\hline Lower Neck (C7/T1) & C7, Suprasternale \\
\hline Upper Lumbar (T12/L1) & T8, T12, C7, Suprasternale \\
\hline Lower Lumbar (L5/S1) & ASIS, PS, PSIS \\
\hline Hip & ASIS, PS, PSIS \\
\hline Shoulder & Acromion, C7, Suprasternale \\
\hline Knee & $\begin{array}{l}\text { Lateral Femoral Condyle (plus Hip } \\
\text { Joint and Lateral Malleolus) }\end{array}$ \\
\hline Elbow & $\begin{array}{l}\text { Lateral Humeral Condyle (plus } \\
\text { Shoulder Joint and Wrist land- } \\
\text { mark) }\end{array}$ \\
\hline Wrist & Wrist \\
\hline Ankle & $\begin{array}{l}\text { Lateral Malleolus (plus Lateral } \\
\text { Femoral Condyle and Hip Joint) }\end{array}$ \\
\hline
\end{tabular}

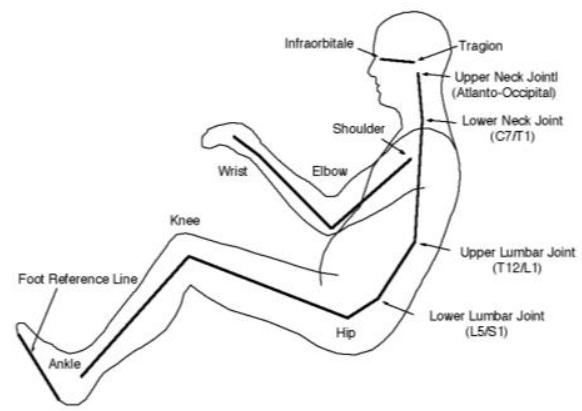

Fig. 4. Internal joints of human body with description [15]

Since the definition of drivers' seat positions are almost always made in relation to their hip locations, the determination of the pelvis geometry is one the main parts of this study. Due to the occurrence of laterally tilted pelvises, the hip joint locations are calculated in three dimensions, then averaged in the XZ plane to obtain a mean hip joint location. The hip joint and lower lumbar joint locations are calculated using the anterior-superior iliac spine (ASIS) landmarks (right and left), the pubic symphysis (PS) landmark, and the posterior-superior iliac spine landmark (PSIS). Seidel [16] defined three pelvis dimensions to further increase accuracy of the location: pelvis width (PW) and pelvis height $(\mathrm{PH})$ and 
pelvis depth (PD). Notably, Seidel also found no important differences between male and female pelvis in these scaling relationships.

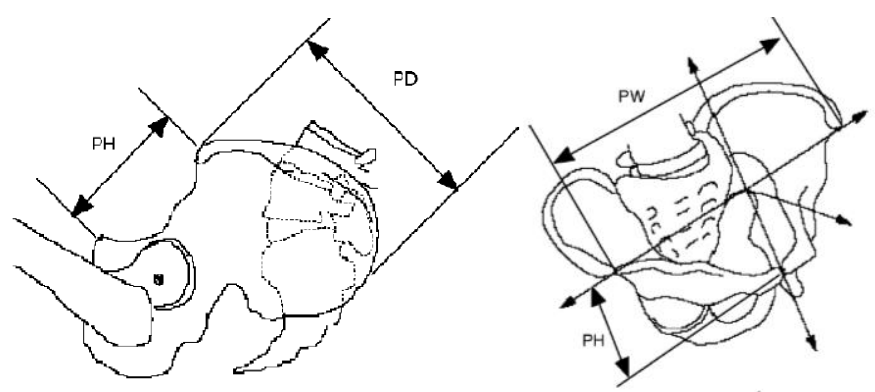

Fig. 5. PW and PH dimensions (left) [15]; PW and PH dimensions (right) [15]
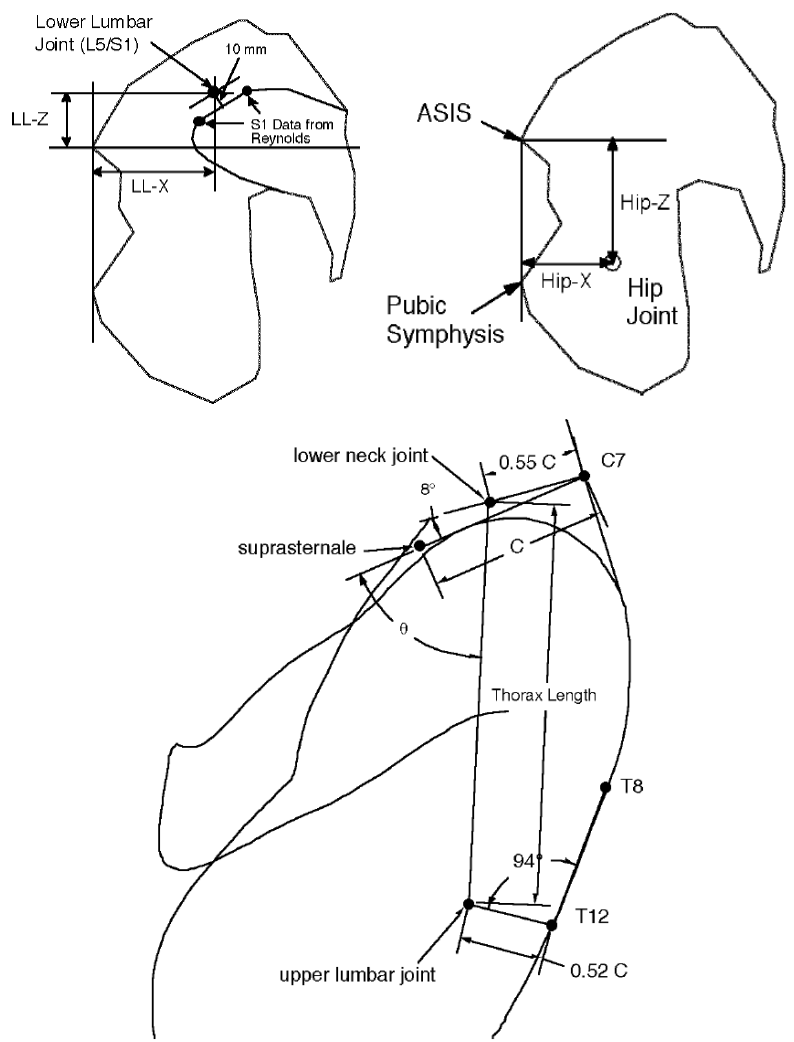

Fig. 6. Lower lumbar joint scaling (left) [15]; hip joint scaling (middle) [15]; upper lumbar and lower neck joint scaling (right) [15]

\subsection{Flesh Margin with BMI and Age Effects}


Recent papers published corrected hip joint estimates based on adiposity of highBMI-value subjects as well as old age [17], [18]. The adjusted calculations shown below were based on the fact that skeletal dimensions are mostly independent of body mass index (unless when referring to those with unusual muscle development, e.g: athletes and professional body builders) as well as the degeneration of bones with age.

The pelvic depth (PD) was adjusted by subtracting the effect of BMI shown in Equation 1. The adjusted depth was calculated as:

$$
\text { PDadj }=\text { PDmin }+ \text { PDmeas }- \text { PDpred }
$$

where PDmin would be the mean expected PD for the subject with the lowest BMI based on the regression given in Equation 2. PDpred is the PD predicted by Equation 2 for the subject accounting for BMI, and PDmeas is the measured PD for the subject.

$$
\text { PDpred }=65.6+4.38 * B M I, R 2=0.43
$$

The bispinous (bi-ASIS) breadth (BB) was adjusted for BMI by subtracting the effect of BMI as shown in Equation 3. (BBpred) was subtracted from the measured bispinous breadth (BBmeas) and the result added to BBmin per Equation 4.

$$
\begin{gathered}
\text { BBadj }=\text { BBmin }+\underset{(3)}{B B m e a s ~}-\text { BBpred } \\
\text { BBpred }=165.3+2.64 * B M I, R 2=0.23
\end{gathered}
$$

The PSIS flesh margin was set to a vector along the pelvic x-axis with a length of $0.0006 * \mathrm{BMI}^{\wedge} 3 \mathrm{~mm}$ based on the adjustment in Reed's [17] study of the ASIS flesh margin. Due to the lack of study at the intimate region of the pubic symphysis, flesh margin at that area will be kept at the $25 \mathrm{~mm}$ estimate.

The hip joint was also adjusted for age as shown by equation 5. LL is length leg measured in millimetres and the origin from which the equation is measured is the midpoint of the biponous (bi-ASIS or PW).

$$
\text { Hip }-Z=-18-0.063 L L-5.403 a g e
$$

\section{Experimental Set-up}

The car chosen to be used as the test vehicle is a 2015 Nissan X-Trail due to long seat tracks and wide opening doors for all subjects to use the apparatus without constriction. 
4 main factors used in experiments: Stature (Height), BMI, Age and Gender (Sex) as recommended from literature review. Sample size of 50 subjects were calculated with a 0.75 power and 0.12 error margin. The subjects are firstly given a survey to fill in.

The subjects are then categorized by the four factors of BMI, age, stature and gender. For stature, an online calculator is used to calculate the percentile of each occupant based on their height. Occupants below 25 th percentile are considered short while those above $75^{\text {th }}$ percentile are considered tall, and the remaining in between are average. For BMI, the occupants are classified according to the World Health Organization (WHO)'s descriptions of body mass index. However, the 'overweight' and 'obese' categories, the were merged due to the lack of participants falling in the obese category, with most of them being relatively close to the overweight limit. For age, occupants are divided to being above and below 50 .

The study is carried out in a few stages. Firstly, the participants are palpated and marked according to the methods previously described. Then, the joint locations of the occupants are marked using the discussed transformations and apparatus like rulers, multi-angle rulers, Vernier callipers and spirit level gauges. Then the experiments are conducted. Participants are asked to assume their most comfortable posture with hands on the steering wheel and right leg on the accelerator (Figure 7). There are 3 parts of the experiment. In the first one, occupants are only allowed to adjust the lumbar supports. Second part is adjustment of the thoracic and sacral supports. Third is adjustment of any and all supports. At the end of each adjustment, the joint angles are measured using a digitalized angle ruler for better accuracy (Figure 8), and the occupants fill in the comfort ratings. Finally, the last parts of the survey are filled in.

Ahead of conducting the experiment, the participants adjusted the seat recline, seat distance, seat height, steering wheel distance and height. The seat recline angle and seat and steering wheel height were fixed after this point. Seat distance and steering wheel distance was allowed to be varied because occupants may feel too constrained after increasing the prominence of the seat apparatus. 


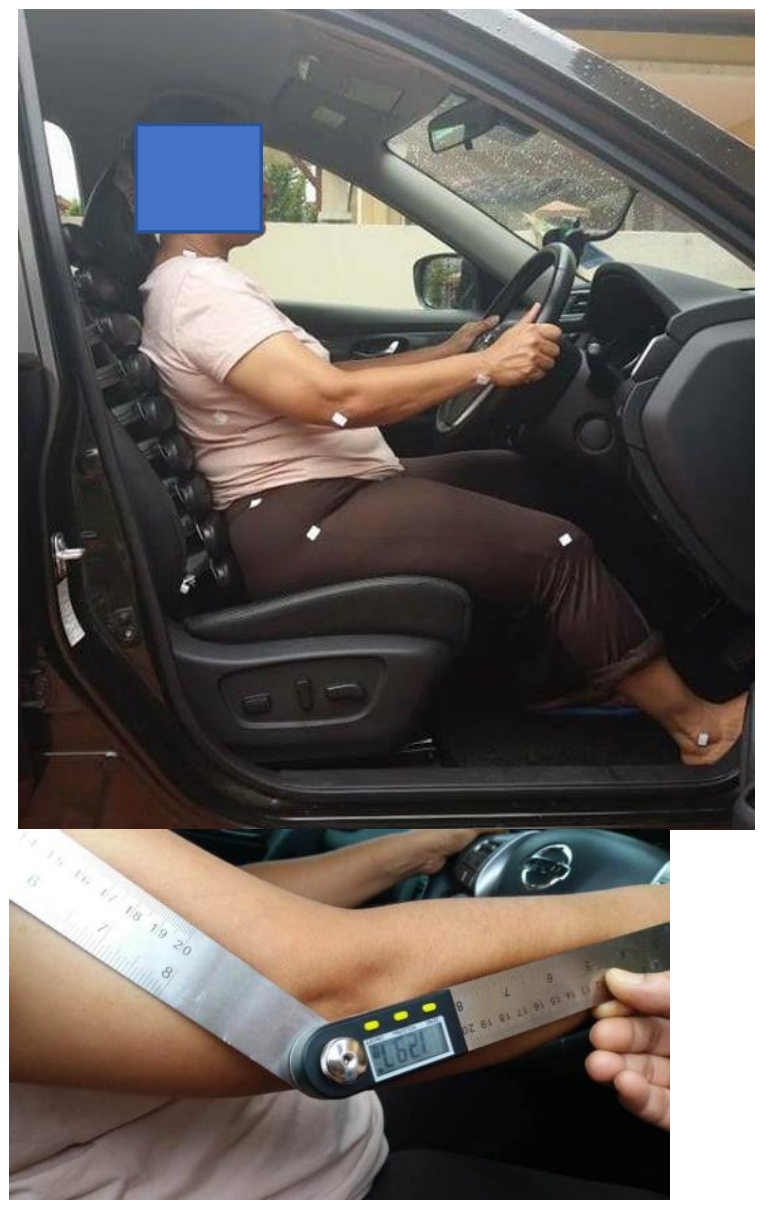

Fig. 7. Subject during experiment with angle measurement method

\section{Result and Discussion}

Of the 50 participants, there were 28 females and 22 males. 12 were short, 22 were average and 16 were tall. 29 are below 50 and 21 are above 50.10 were underweight, 23 were normal weight and 17 were overweight/obese. Angle values of this study are compared to 12 other studies conducted between this year and 1998 in Figures 10-14 below. Aside from previous studies, the measured values are also compared to predetermined range of motion values. The comparison is necessary to ensure the angles measured from this study are reasonable within human capability and preference, and sustainable in future researches. More importantly, comparison will ensure the method and apparatus used is somewhat accurate. 


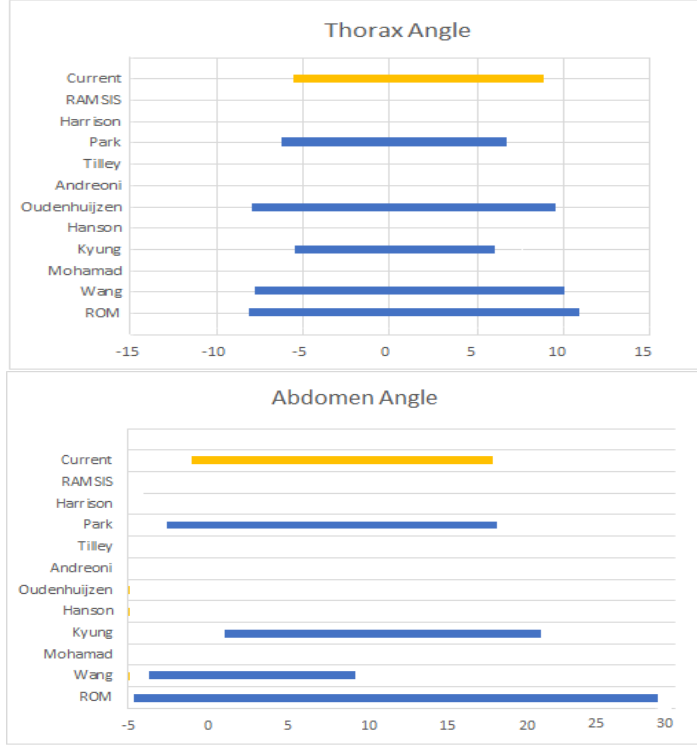

a) Thorax comparison

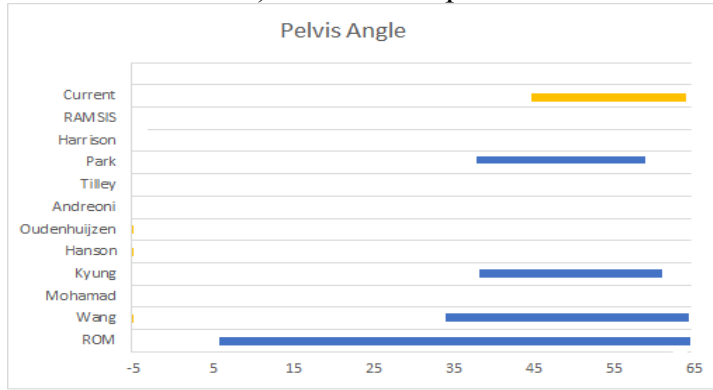

Elbow Angle

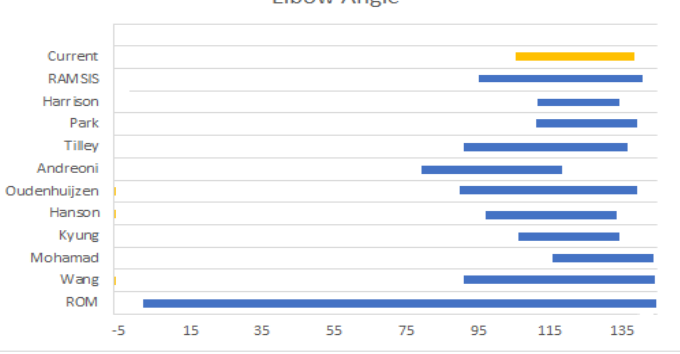

c) Pelvis comparison

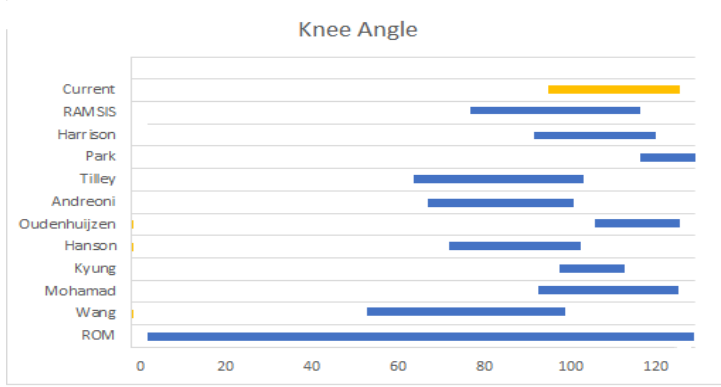

b) Abdomen comparison

d) Elbow comparison 


\section{e) Knee comparison}

Fig. 8. Range of angles for a) Thorax, b) abdomen, c) Pelvis, d) elbow and e) knee preferred and comparison with literatures

Firstly, all angles are verified to be within acceptable ROM, so the apparatus was confirmed to be reliable. Moving on from that, the measured data was compared to previous studies, and some differences were noted. Due to the complexity of measuring posture and the interdependence of joint angles, the main problem in comparing different studies is that the results are influenced by the heterogeneity of experimental settings. Factors like data type (2D or 3D), number of participants, number of joints, recording time, symmetry, experimental method (field of laboratory), car type (sedan, SUV, etc.), study region, optimum vs. preferred, questionnaires, measurement method and reference point (H-point or Seat Reference Point).

One-Way Repeated Measures ANOVA was performed to test for difference in the 5 angles between each experiment. The null hypotheses of this ANOVA test states that there are no differences in the means of angles between the three seat configurations. The alternate hypotheses state that there are differences in the means of angles between the three seat configurations. The different seat configurations did not lead to any statistically significant changes in the elbow angle, $F(2,48)=1.503, p=.235$. or in the knee angle $F(2,48)=$ $1.218, p=.307$. The null hypothesis is accepted for these two cases. This would mean that regardless of the seat configuration, participants did not alter their knee and elbow angles, possibly due to constricts from the task of driving. However, there were statistically significant differences in the thorax angle $F(1.545,39.353)=5.138, p=.045$, as well as in the abdomen angle $F(1.505,38.591)=17.096, p=.000$ and the pelvis angle $F(1.693$, $42.170)=3.466, p=.042$. For these cases, the null hypothesis is rejected and the alternate hypothesis is accepted.

To identify the human factors and its interactions' effects on each joint, Factorial ANOVA was carried out. It is important to understand how Factorial ANOVA works in SPSS. The analysis essentially tests for the variance between means. In this software, the analysis chooses one standard mean and compares all the rest of the means to it. The default settings are to choose the combination of the highest numerical coded factors. Categories are coded as seen in Table 3:

Table 1. Coding for each category

\begin{tabular}{|l|l|l|l|}
\hline Stature & 3 - Average & 2 - Short & 1 - Tall \\
\hline BMI & 3 - Normal & 2 - Underweight & $1-$ Overweight/Obese \\
\hline Age & & 2 - Below 50 & $1-$ Above 50 \\
\hline Gender & & 2 - Male & $1-$ Female \\
\hline
\end{tabular}


This is to set the standard as a normal BMI, male, below 50 with average stature. Every mean angle of all other categories in any combination is compared to this standard. The ANOVA analysis is then performed with the null hypothesis that the mean of all other combination of categories are equal to the mean of the standard. The parameter estimates in Table 4 tell us which specific category combinations are most influential on the angles. The intercept below is the standard mean angle of a normal BMI, male, below 50 with average stature

Table 2. Combination of Parameter estimates on different body parts

Thorax

\begin{tabular}{|l|r|r|r|r|}
\hline \multicolumn{1}{|c|}{ Parameter } & \multicolumn{1}{c|}{ B } & \multicolumn{1}{c|}{$\begin{array}{c}\text { Std. } \\
\text { Error }\end{array}$} & \multicolumn{1}{c|}{$\begin{array}{c}\text { Sig. } \\
\text { level }\end{array}$} & \multicolumn{1}{c|}{$\begin{array}{c}\text { Observed } \\
\text { Power }\end{array}$} \\
\hline Intercept & 11.0 & 0.222 & 0.000 & 0.738 \\
\hline$(\mathrm{BMI}=1)$ & +14.0 & 4.184 & 0.020 & 0.514 \\
\hline$($ Gender $=2) *(\mathrm{BMI}=1)$ & +16.0 & 3.254 & 0.018 & 0.582 \\
\hline$($ Age $=1) *($ Gender $=1)$ & +9.0 & 0.360 & 0.031 & 0.640 \\
\hline$($ Age $=1) *($ Stature $=2)$ & -10.0 & 0.252 & 0.028 & 0.715 \\
\hline$($ Stature $=1) *($ BMI $=1)$ & +13.0 & 3.895 & 0.023 & 0.534 \\
\hline$($ Stature $=1) *($ Gender $=1) *($ Age $=1)$ & -9.0 & 0.428 & 0.031 & 0.685 \\
\hline
\end{tabular}

Abdomen

\begin{tabular}{|l|r|r|r|r|}
\hline \multicolumn{1}{|c|}{ Parameter } & \multicolumn{1}{c|}{ B } & \multicolumn{1}{c|}{$\begin{array}{c}\text { Std. } \\
\text { Error }\end{array}$} & \multicolumn{1}{c|}{$\begin{array}{c}\text { Sig. } \\
\text { level }\end{array}$} & \multicolumn{1}{c|}{$\begin{array}{c}\text { Observed } \\
\text { Power }\end{array}$} \\
\hline Intercept & 23.0 & 0.141 & 0.000 & 0.749 \\
\hline$($ Age $=1)$ & -19.0 & 0.190 & 0.038 & 0.725 \\
\hline$($ Gender $=2) *(\mathrm{BMI}=1)$ & -17.0 & 5.125 & 0.032 & 0.599 \\
\hline$($ Age $=1) *($ Gender $=1)$ & -21.0 & 0.235 & 0.040 & 0.692 \\
\hline$($ Stature $=1) *(\mathrm{BMI}=1)$ & -24.0 & 4.605 & 0.046 & 0.612 \\
\hline$($ Stature $=1) *($ Gender $=1) *($ Age $=1)$ & -25.0 & 0.360 & 0.008 & 0.677 \\
\hline
\end{tabular}

Pelvis

\begin{tabular}{|l|c|c|c|c|} 
Parameter & B & Std. & Sig. level & Observed \\
\hline
\end{tabular}




\begin{tabular}{|l|r|r|r|r|}
\hline & & \multicolumn{1}{|c|}{ Error } & & \multicolumn{1}{|c|}{ Power } \\
\hline Intercept & 41.0 & 0.192 & 0.000 & 0.741 \\
\hline$(\mathrm{BMI}=1)$ & +12.0 & 6.420 & 0.028 & 0.489 \\
\hline$($ Gender $=2) *(\mathrm{BMI}=1)$ & +18.0 & 8.214 & 0.009 & 0.475 \\
\hline$($ Gender $=1) *(\mathrm{BMI}=1)$ & +9.0 & 6.104 & 0.031 & 0.501 \\
\hline
\end{tabular}

Elbow

\begin{tabular}{|l|r|r|r|r|}
\hline \multicolumn{1}{|c|}{ Parameter } & \multicolumn{1}{c|}{ B } & \multicolumn{1}{c|}{$\begin{array}{c}\text { Std. } \\
\text { Error }\end{array}$} & \multicolumn{1}{c|}{$\begin{array}{c}\text { Sig. } \\
\text { level }\end{array}$} & $\begin{array}{c}\text { Observed } \\
\text { Power }\end{array}$ \\
\hline Intercept & 114.0 & 0.184 & 0.000 & 0.748 \\
\hline$($ Age $=1)$ & -11.0 & 0.345 & 0.022 & 0.692 \\
\hline$($ Stature $=1)$ & -9.0 & 0.241 & 0.034 & 0.710 \\
\hline$($ Stature $=2)$ & +16.0 & 0.219 & 0.018 & 0.721 \\
\hline
\end{tabular}

Knee

\begin{tabular}{|l|r|r|r|r|}
\hline \multicolumn{1}{|c|}{ Parameter } & \multicolumn{1}{c|}{ B } & \multicolumn{1}{c|}{$\begin{array}{c}\text { Std. } \\
\text { Error }\end{array}$} & \multicolumn{1}{c|}{$\begin{array}{c}\text { Sig. } \\
\text { level }\end{array}$} & $\begin{array}{c}\text { Observed } \\
\text { Power }\end{array}$ \\
\hline Intercept & 129.0 & 0.202 & 0.000 & 0.742 \\
\hline$(\mathrm{BMI}=1)$ & -23.0 & 0.451 & 0.013 & 0.652 \\
\hline$($ Gender $=1) *(\mathrm{BMI}=1)$ & +10.0 & 0.520 & 0.024 & 0.640 \\
\hline
\end{tabular}

By studying the perimeter estimates, we can tell which interactions or main factors caused the biggest deviation from the standard mean. In the thorax angle, the interaction of male and high BMI caused the biggest change in angle, at an estimated mean of $26.0^{\circ}$. In the abdomen, the biggest effect was from a tall, older female making the angle $-2.0^{\circ}$ In the pelvis, it was the interaction of male with high BMI, causing a $59.0^{\circ}$ angle. In the elbow, it was the from the short occupants causing a $130.0^{\circ}$ angle. Finally, in the occupants with high BMI, causing an angle of $106.0^{\circ}$. There are larger errors in some of the mean angles, like the pelvis and abdomen, where the joints used are covered with a lot of fat and harder to palpation and predict. 


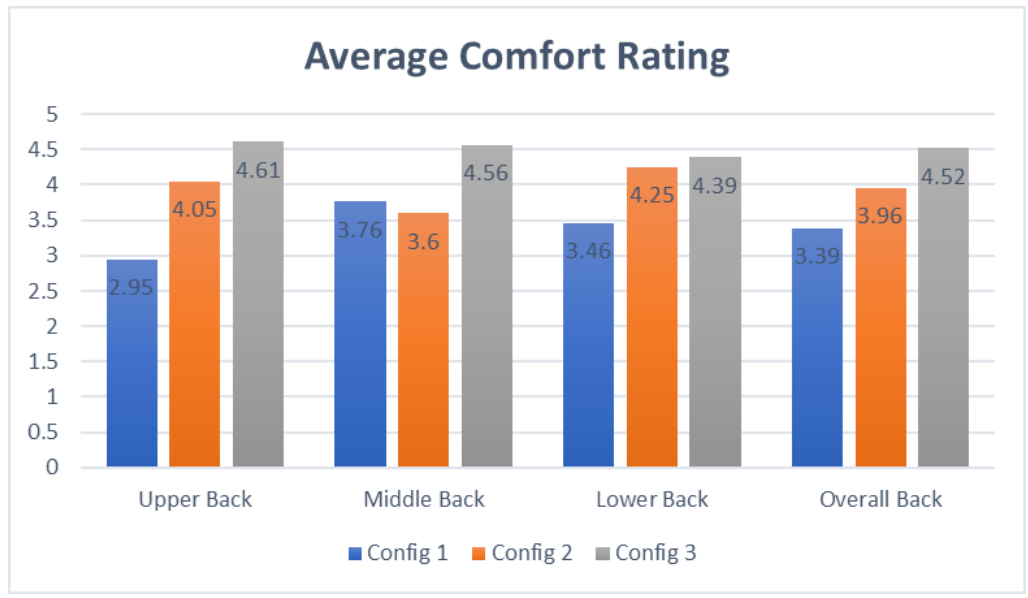

Fig. 9. Graph of Comfort Ratings

The comfort rating survey divided the back into 3 segments: upper, middle and lower. Ratings were on a scale of $1-5,1$ being 'Uncomfortable' and 5 being 'Comfortable'. The overall back comfort is calculated as the average comfort of all three areas. From the graph in Figure 15, there was a definitely increase in all three segments and the overall back in the last experiment of the seat that had all supports utilized. In the first experiments, occupants felt very little comfort in the upper back and lower back, though the middle was relatively higher. This could be due to the lumbar support only allowing occupants to have support in that region and not in the upper and lower. It could be possible that occupants had to compromise one for the other. In the second experiments, with sacral and thoracic support, most occupants had better overall back comfort, though they were some who felt the middle part was now lacking. Finally, in the last experiments, all three segments were the highest with the highest overall back rating. It is clear that there is a definite different in the comfort felt by occupants in their true preferred posture compared to ones that they had less adjustability in.

\section{Conclusion}

All the objectives of the experiment were met. The apparatus was able to allow occupants to define their preferred posture in a realistic setting with accuracy. The incorporation of the apparatus was proven to cause changes in postural angles that positively affected the static comfort of occupants. It was also identified that human factors affect the joint angles in a variety of ways, and have different influences on different joints. A limitation that may have potentially influenced the accuracy of the results is the palpation and determination of joints that may not have been very accurate. For future work, a more advanced apparatus could be created using similar concept that will allow more adjustments (support tilt, height variation, etc.) and have lesser influence over the subject's comfort perception. The angles recorded in this study can be used to verify predicted posture from Digital Human Models in the future, as well as benchmark data for similar experimental set-ups. More objective 
measures like EMG, pressure mapping and vibration analysis can be done as extensions to further validate the efficiency of the sacral + thoracic support.

\section{References}

1 D. Mohamad, B.M.Deros, D.A. Wahab, D.D.I. Daruis and A.R. Ismail, "Integration of Comfort into a Driver' s Car Seat Design Using Image Analysis Darliana Mohamad",American Journal of Applied Science, vol. 7, no. 7, pp. 937-942, 2010.

2 M. P. De Looze, L. F. M. Kuijt-Evers, and J. Van Dieën, "Sitting comfort and discomfort and the relationships with objective measures," Ergonomics, vol. 46, no. 10, pp. 985-997, 2003.

3 M. P. Reed, L. W. Schneider, and L. L. Ricci, "Survey of Auto Seat Design Recommendations for Improved Comfort," no. April, pp. 1-96, 1994.

4 B. M. Deros et al., "ScienceDirect Incorporating Malaysian's Population Anthropometry Data in the Design of an Ergonomic Driver's Seat," Procedia - Soc. Behav. Sci., vol. 195, no. 195, pp. 2753-2760, 2015.

5 M. P. Reed, M. B. Parkinson, and D. B. Chaffin, "A New Approach to Modeling Driver Reach," Sae Tech. Pap. Ser., no. 724, 2003.

6 S. A. Ziolek, "Beyond Percentiles : An Examination of Occupant Anthropometry and Seat Design," Hum. Factors, no. 724, 2004.

7 T. Seitz and H. Bubb, "SAE TECHNICAL Measuring of Human Anthropometry, Posture and Motion," no. 724, 1999.

8 B. Deros et al., "Advances in Natural and Applied Sciences Incorporating Anthropometrics Data in Designing Driver' s Seat for Malaysian Made Compact Cars," vol. 8, no. June, pp. 915-919, 2014.

9 M. D. Stinson, A. Porter-Armstrong, and P. Eakin, "Seat-interface pressure: A pilot study of the relationship to gender, body mass index, and seating position," Arch. Phys. Med. Rehabil., vol. 84, no. 3 SUPPL. 1, pp. 405-409, 2003.

10 G. Andreoni, G. C. Santambrogio, M. Rabuffetti, and A. Pedotti, "Method for the analysis of posture and interface pressure of car drivers," Appl. Ergon., vol. 33, no. 6, pp. 511-522, 2002.

11 M. J. Griffin, "Whole-Body Vibration,” Encycl. Vib., pp. 1570-1578, 2001.

12 H. Nahvi, M. H. Fouladi, and M. J. Mohd Nor, "Evaluation of whole-body vibration and ride comfort in a passenger car," Int. J. Acoust. Vib., vol. 14, no. 3, pp. 143-149, 2009.

13 T. R. Bush, F. T. Mills, K. Thakurta, R. P. Hubbard, and J. Vorro, "The Use of Electromyography for Seat Assessment and Comfort Evaluation," Soc. Automot. Eng., no. 412, pp. 143-1995, 1995.

14 S. J. Park and B. Min, "Development of the Evaluating System for Ride Comfort and Fatigue in Vehicle," SAE Tech. Pap., no. 724, 2013.

15 M. P. Reed, M. A. Manary, and L. W. Schneider, "Methods for Measuring and Representing Automotive Occupant Posture," Soc. Automot. Eng., no. 724, 1999.

16 G. K. Seidel, D. M. Marchinda, M. Dijkers, and R. W. Soutas-Little, "Hip joint center location from palpable bony landmarks-A cadaver study," J. Biomech., vol. 28, no. 8, pp. 995-998, 1995. 
17 M. P. Reed, S. M. Ebert-Hamilton, and J. D. Rupp, "Effects of Obesity on Seat Belt Fit," Traffic Inj. Prev., vol. 13, no. 4, pp. 364-372, 2012.

18 R. Hara, J. McGinley, C. Briggs, R. Baker, and M. Sangeux, "Predicting the location of the hip joint centres, impact of age group and sex," Sci. Rep., vol. 6, no. 1, p. 37707, 2016. 